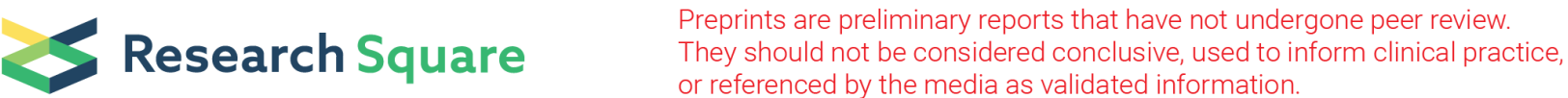

\section{Comparative Study of Preoperative Scores to Predict Lymph Node Metastasis in Patients With Gastric and Colon Cancers: A Case Control Study}

\section{Xiangjian Zheng ( $\nabla 07105212 @ q q . c o m)$}

The Dingli Clinical Institute of Wenzhou Medical University,Wenzhou,Zhejiang,China https://orcid.org/0000-0002-3605-4946

\section{Xiaodong Chen}

The Second Affiliated Hospital of Wenzhou Medical University,Wenzhou,Zhejiang,China

Min Li

The Dingli Clinical Institute of Wenzhou Medical University,Wenzhou,Zhejiang,China

\section{Chunmeng Li}

The Dingli Clinical Institute of Wenzhou Medical University,Wenzhou,Zhejiang,China

\section{Xian Shen}

The Second Affiliated Hospital of Wenzhou Medical University,Wenzhou,Zhejiang,China

\section{Research}

Keywords: lymph node metastasis, gastric and colon cancers, preoperative scores, ROC curve

Posted Date: June 30th, 2020

DOl: https://doi.org/10.21203/rs.3.rs-37182/v1

License: (c) (1) This work is licensed under a Creative Commons Attribution 4.0 International License. Read Full License 


\section{Abstract}

Background:Surgery combined with chemo-radiotherapy is a recognized model for the treatment of gastric and colon cancers. Lymph node metastasis determines the patient's surgical or comprehensive treatment plan. The purpose of this study was to compare preoperative scores to better predict lymph node metastasis in patients with gastric and colon cancers.

Methods:A total of 768 patients with gastric cancer (312) and colon cancer (462) were enrolled in our study. Preoperative serum markers, immune markers, and clinical tumor characteristics were evaluated by single-factor analysis. Logistic analysis was used to identify independent predictors of lymph node metastasis in patients with gastric and colon cancers. These independent risk factors were integrated into preoperative scores, which was evaluated by receiver operating characteristic (ROC) curves.

Results:The result showed that serum markers (CA125, hemoglobin, albumin), immune markers (S100, CD31, d2-40), and tumor characteristics (pathological type, tumor size) were independent risk factors for lymph node metastasis in patients with gastric and colon cancers. The preoperative scores reliably predicted lymph node metastasis in patients with gastric and colon cancers with a higher area under the ROC curve (0.901). Compared to the other independent risk factors, the area under the ROC curve of this indicator was 0.923 and 0.870 , for gastric and colon cancers, respectively. Based on the ROC curve, the ideal cutoff value of preoperative scores to predict lymph node metastasis was established to be 287 .

Conclusion: The preoperative scores is a useful indicator that could be used to predict lymph node metastasis in patients with gastric and colon cancers.

\section{Introduction}

Gastric cancer and colon cancer are the two most common malignant tumors of the digestive tract occupying the third and second place, respectively, in cancer-related mortalities[1, 2]. Surgical intervention continues to be the most effective way to treat gastric and colon cancers. However, the rate of postoperative cancer recurrence is high, and the long-term survival rate in patients is low. Lymph node metastasis is a common pattern observed in the advanced stages of cancer and a significant cause of death. More than $50 \%$ of gastric and colon cancer patients have lymph node metastases at the time of diagnosis, which results in a 5-year survival rate of less than $30 \%$ [3].

At present, surgery combined with chemo-radiotherapy is a recognized model for the treatment of some gastric and colon cancers. Lymph node metastasis is the basis for clinical tumor staging. It determines the patient's surgical or comprehensive treatment plan. The degree of metastasis is an indicator, which plays an essential role in designing an effective treatment and reducing trauma, which in turn, could considerably improve the patient's prognosis [4]. Therefore, accurate preoperative prediction of lymph node metastasis is crucial. 
In recent years, with the steady development of radiographic-detection technology, new imaging diagnostic tools have gradually attracted the attention of clinicians. However, the sensitivity and specificity of techniques such as computed tomography (CT) and magnetic resonance imaging (MRI) to scan lymph nodes with a diameter of less than $1 \mathrm{~cm}$ are still low [5].Thus, these diagnostic tools are often of limited use in such patients. Some studies have shown that positron-emission tomography integrated with computed tomography (PET-CT) can be an independent predictor of lymph node metastasis in gastric cancers. But the scope of application is limited because of its high false-positive rate and cost [6].

The study of preoperative serum indicators to predict lymph node metastases has also been widely reported. These are similar to serum tumor markers such as CA125, CEA, and CA199, but have low sensitivity and specificity. Some researchers have tried to improve its predictive value by combining several serum tumor markers [7]. The neutrophil to lymphocyte (NLR) and platelet to lymphocyte ratios (PLR) are crucial prognostic factors in the treatment of different types of cancers [8]. However, the value of these preoperative serum markers in diagnosing lymph node metastasis alone is limited. With the development of tumor immunology, the relationship between the clinicopathologic features of tumors and the extent of lymph node metastasis in patients with gastric and colon cancers is gradually being recognized. Till date, few study has been reported on the combination of preoperative serum markers and clinicopathologic features to predict lymph node metastasis.

Therefore, the purpose of this study was to better predict lymph node metastasis in patients with gastric and colon cancers and to help improve the accuracy of preoperative diagnoses. We compared the preoperative scores in patients who underwent surgery. We found that the preoperative scores was helpful and reliable in predicting lymph node metastasis in patients with gastric and colon cancers. An individualized multimodality treatment could thus be provided to these patients.

\section{Materials And Methods}

\section{Patients}

This paper retrospectively analyzed the data of 833 patients with gastric and colon cancers who underwent gastric and colon cancer surgery in The Dingli Clinical Institute of Wenzhou Medical University (Wenzhou Central Hospital) from January 2010 to January 2018. The following information was collected and recorded: the patient's personal information (age, sex, body mass index, family history), preoperative serum markers (tumor markers, routine blood index, albumin), tumor characteristics (location, size, pathological type, histopathological differentiation, lymph node metastasis), and immunology indices (CD31, MRP3, EGFR, P53, CDX2). Histopathological differentiation was classified as "well-differentiated" (moderately or highly differentiated) and "poorly differentiated" (poorly differentiated or undifferentiated) tumors. The pathological types are divided into ulcer and non-ulcer types. All patients were examined by preoperative histologic examination. Exclusion criteria were as follows: (1) history of other malignancies (10 cases), (2) history of gastrointestinal resections (6 cases), (3) history of distant metastases (9 cases), (4) history of preoperative chemo-radiotherapy (15 cases), (5) history of liver 
diseases such as cirrhosis (8 cases), (6) history of coagulation disorders and autoimmune diseases (11 cases), and (7) history of serious hematologic diseases ( 6 cases).Consequently, 768 patients were enrolled. This study was approved by the committee of The Dingli Clinical Institute of Wenzhou Medical University (Wenzhou Central Hospital). All patients were informed and they signed the informed consent.

\section{Diagnosis of lymph node metastasis}

According to the pathological results of the preoperative endoscopic biopsy, intraoperative frozen sections and postoperative pathological findings of the patient were used to diagnose lymph node metastasis.

\section{The cutoff point of the preoperative scores}

We plotted the receiver operating characteristic (ROC) curve and used the maximum value of the Youden index as the cutoff value for the preoperative scores. Subsequently, the patients were divided into two groups according to the cutoff point, for further study.

\section{Statistical analysis}

We performed the Kolmogorov-Smirnov test to determine the normality of continuous parameters such as neutrophil count, lymphocyte count, platelet count, monocyte count, PLR, and NLR. The mean and standard deviation values were used for the normal distributed data, whereas the median and interquartile range values were used for the non-normal distributed data. The Mann-Whitney Utest was used to compare the non-normal distributed variables between the lymph node metastasis group and the non-lymph node metastasis group. An ROC analysis was conducted to determine the performance of the variables. The relationship between clinicopathologic characteristics and the preoperative scores was analyzed using the $\chi 2$ test. In addition, the $\chi 2$ test was used for the univariate analysis of lymph node metastasis. Based on the results of this analysis, a multivariate logistic regression analysis was used to calculate the odds ratio (OR) and $95 \%$ confidence interval (Cl) of the confirmed independent variables. The area under the ROC curve (AUC) was used to compare the indicator to other clinicopathologic characteristics. A value of $\mathrm{P}<0.05$ was considered statistically significant. Statistical analyses were performed using SPSS software (version 21.0; SPSS Inc., Chicago, IL, USA).

\section{Results}

\section{Patient characteristics}

As shown in Table 1, among the 768 patients, 510 were males, and 258 were females. The median age of the patients was 66 years, and the quartile ranged from 58 to 75 years. The average tumor size of the patients was $4.12 \mathrm{~cm}$. There were 312 cases of gastric cancer and 462 cases of colon cancer. The majority (531 cases) were at stage T3/4. According to the pathological type, the majority $(73.4 \%$ ) of patients with cancers were classified as ulcerative. Based on histopathological findings, the patients had "well-differentiated" (573) and "poorly differentiated" tumors (195). Lymph node metastasis had occurred in 384 patients ( 6 of whom had lost clinical data). 


\section{Characteristics of preoperative serum markers in patients with gastric and colon cancer with lymph node metastasis}

As shown in Table 2, CA125, neutrophils, NLR, and PLR were significantly higher in patients with gastric and colon cancers with lymph node metastasis than those without $(P<0.05)$. In contrast, patients with gastric and colon cancers without lymph node metastasis had higher hemoglobin levels, erythrocyte numbers, and albumin values $(P<0.05)$. The ROC curve was used to further analyze the significantly different variables. As shown in Fig 1, the AUC areas of CA125 $(0.602,95 \% \mathrm{Cl} 0.529-0.675)$ and hemoglobin $(0.618,95 \% \mathrm{Cl} 0.546-0.691)$ were larger, but their sensitivity and specificity were still low.

\section{Univariate analysis of clinicopathologic characteristics}

The $\chi 2$ test was used to examine the relationship between clinicopathologic features and lymph node metastasis in patients with gastric and colon cancers. As shown in Table 3 , tumor size $(P=0.012)$, pathological type $(P=0.000)$, histopathological differentiation $(P=0.000)$, depth of invasion $(P=0.000)$, $\mathrm{S} 100(P=0.000)$, and $C D 31 / \mathrm{d} 2-40(P=0.000)$ were significantly different in patients with gastric and colon cancers with or without lymph node metastasis. In contrast, there were no significant differences in body mass index, gender, age, or BMI between the two groups. Among these parameters, S100 positive indicates nerve infiltration, while CD31/ d2-40 positive indicates vascular infiltration.

\section{Multivariate analysis of lymph node metastasis}

Logistic multivariate regression analysis was used to examine independent risk factors for lymph node metastasis. As shown in Table 4, the risk of lymph node metastasis in patients with gastric cancer was significantly associated with albumin $(O R=7.804, P=0.006)$, histopathological differentiation ( $O R=3.601$, $\mathrm{P}=0.066)$, depth of invasion ( $\mathrm{OR}=5.982, \mathrm{P}=0.008)$, and vascular infiltration ( $\mathrm{OR}=29.251, \mathrm{P}=0.000)$. Colon cancer patients were significantly associated with $\mathrm{CA} 125(\mathrm{OR}=2.660, \mathrm{P}=0.032), \mathrm{PLR}(\mathrm{OR}=3.896, \mathrm{P}=0.008)$, vascular infiltration $(\mathrm{OR}=9.177, \mathrm{P}=0.000)$, and nerve infiltration $(\mathrm{OR}=8.103, \mathrm{P}=0.001)$. Thus, independent risk factors were different in the two groups. Integrating these two patient groups, as shown in Table 5 , indicated significant association with $\mathrm{CA} 125(\mathrm{OR}=2.851, \mathrm{P}=0.009)$, albumin $(\mathrm{OR}=2.334, \mathrm{P}=0.050)$, hemoglobin $(\mathrm{OR}=3.255, \mathrm{P}=0.008)$, pathological type $(\mathrm{OR}=2.828, \mathrm{P}=0.021)$, tumor size $(\mathrm{OR}=15.847$, $P=0.015)$, vascular infiltration $(O R=18.200, P=0.000)$, and nerve infiltration $(O R=2.812, P=0.016)$. From this data, we can derive independent risk factors for gastric and colon cancers.

\section{Construction of the preoperative scores for lymph node metastasis in gastric and colon cancers}

According to multivariate logistic regression analysis, independent risk factors were selected. As shown in Table 5, the preoperative score obtained from the logarithmic conversion of each independent risk factor multiplied by 100 was calculated and assigned to each element. The preoperative scores was established by summing up the calculated results and marked as Score. ROC curve analysis was used to further determine whether the preoperative scores had a high accuracy in predicting lymph node metastasis in gastric and colon cancers. As shown in Figure 2, the area under the ROC curve was 0.901 in gastric and 
colon cancer patients. Compared to other independent risk factors for gastric cancer and colon cancer, as shown in Figures 3 and 4 , the AUC values were $(0.923,95 \% \mathrm{Cl} 0.866-0.980)$ and $(0.870,95 \% \mathrm{Cl} 0.813-$ 0926) respectively, which were higher. Therefore, it was more reliable as a predictor of lymph node metastasis in patients with gastric cancer and colon cancer.

\section{The relationship between preoperative scores and different clinicopathologic characteristics in gastric and colon cancer patients}

According to the ROC curve, the cutoff value of the preoperative scores for lymph node metastasis was 287. The specificity and sensitivity of this diagnosis were $88.1 \%$ and $75 \%$, respectively. Based on the cutoff value, the gastric and colon cancer patients were divided into "high Score group ( $\geq 287)$ " and "low Score group (<287)." Among the enrolled patients, the high Score group accounted for $43.7 \%$. As shown in Table 6, according to the clinicopathological characteristics of our study, a higher score was associated with a larger tumor size, a more severe pathological cancer type, a higher degree of differentiation, a deeper degree of invasion, a higher invasion range, and a higher rate of lymph node metastasis. This finding suggests that the preoperative scores is a stronger predictor of lymph node metastasis in patients with gastric and colon cancers than other independent indicators. Our results also suggest that using the numerical value 287 as the cutoff point can provide more effective information.

\section{Discussion}

Lymph node metastasis is the most common metastatic model of gastric and colon cancers. With the development of surgery in the management of these cancers, laparoscopic surgery, by virtue of its shortterm minimally invasive advantages and long-term safety, has been confirmed as an early alternative for gastric and colon cancers by several clinical randomized controlled trials and meta-analyses $[9,10]$. However, in the case of advanced tumors, the use of laparoscopy is still controversial [11]. In addition, MAGIC, FNCLCC, and FFCD studies ${ }^{12,13}$ report that preoperative chemotherapy can not only play a role in reducing the period, but also prolong survival. Therefore, preoperative evaluation of lymph node metastasis is crucial in improving the surgical outcomes and reducing the extent of damage caused to patients.

Currently, clinical laparoscopy and pathological biopsy are the gold standards for treatment, however, the degree of trauma caused by these procedures render them less desirable. Therefore, non-invasive diagnosis models have a good scope for further exploration. CT and MRI are the most common techniques used to predict lymph node metastases. However, the sensitivity of CT and MRI was only 50\% in the determination of metastasis of lymph nodes that were $5 \mathrm{~mm}$ or smaller [5]. While Pet-CT can assess the involvement of lymph nodes and distant organs in tumor patients [14], its high cost outweighs its popularity. Therefore, there is an urgent need to develop a more effective method for the preoperative diagnosis of lymph node metastasis in patients with gastric and colon cancers. 
Preoperative serum markers are commonly used in predicting lymph node metastasis of gastrointestinal tumors. In the current treatment scenario, serum tumor markers are most commonly used, but they have not been widely adopted owing to their low sensitivity and specificity. Some researchers [7] use a combination of several markers to improve the sensitivity of diagnoses $(96.3 \%)$ and specificities $(69.8 \%)$, however, there are only few such clinical studies that are relevant. In addition, increasing attention has been paid to the relationship between a tumor and the body's inflammatory response [15].Chronic inflammatory response induces lymphocyte to infiltrate into the tumor, and consequently, into the surrounding tissues, thus increasing the chances of metastases. Studies have shown that PLR and NLR contribute to the diagnoses and prognoses of malignant tumors [16]. In our study, CA125 (OR = 2.660, P = $0.032)$ and PLR $(O R=3.896, P=0.008)$ were independent risk factors for lymph node metastasis in patients with colon cancer, but not for gastric cancer. Apparently, these variables could not be used exclusively in predicting lymph node metastasis in all patients.

With the development and use of immunohistochemistry, it is possible to predict the metastasis of lymph node tumors more accurately. Li found that the metastasis rate was higher in patients with T4 stage gastric cancer, with a lower degree of differentiation, and Borrmann type $\nabla+\mathbb{Q}$ [17]. In addition, a study [18] found that the gross type, pathological grade, and invasion depth were risk factors for lymph node metastasis in colon cancer. These clinicopathological features can be confirmed by preoperative endoscopic examinations. Therefore, we attempted to analyze the relationship between clinicopathologic features and lymph node metastasis in gastric and colon cancers.

Scartozzi [19] studied 734 patients with gastric cancer and showed that a positive rate of nerve infiltration ( $\mathrm{NI}$ ) and vascular infiltration $(\mathrm{VI})$ was $26 \%$, which is associated with lymph node metastasis. In our study, the values for $\mathrm{NI}$ and VI were $35.43 \%$ and $39.76 \%$, respectively. We used HE and S100, D2-40, and CD31 protein immunohistochemical staining to improve the accuracy of $\mathrm{NI}$ and VI. The effect of $\mathrm{VI}$ on gastric cancer and colon cancer has been studied [20,21]. But the effect of NI presented different sounds. Although the relationship between $\mathrm{NI}$ and prognosis has been evaluated by meta-analysis of colon cancer studies [22],this relationship remains controversial. A meta-analysis [23] included a total of 30590 patients with gastric cancer in 24 studies and found that the $\mathrm{NI}$ positive rate was $6.8-75.6 \%$, with an average positive rate of $40.9 \%$. The difference in NI positive rate in each study is large, which is mainly due to the difference in the definition of $\mathrm{Nl}$ in each study. Currently, there is still no unified standard for the definition of NI. Tanaka reports [24] that NI has a prognostic value in patients with a T2 grade gastric tumor, but not in patients with grade T3/4 tumor. Another study [25]reports that NI has no statistical significance in cardiac cancers. Our study too, found that NI was not an independent risk factor for gastric cancer.

Ren [26] reports that tumor invasion depth is an independent risk factor for lymph node metastasis in gastric cancers. A study by Bravo[27] indicates that the risk of lymph node metastasis by tumor infiltration into the submucosa is 3.103 times higher than that of the tumor confined to the mucosa. We obtained similar results in our study, with the only difference being the type of cancer evaluated. These findings can be attributed to the abundant lymphatic vascular network in the gastric submucosa and the 
higher concentration of lymphatic reflux channels in the serosal layer. In addition, it is seen in Kodera's study[28] that the lymph node metastasis rate for patients with well-differentiated gastric cancer is $6.9 \%$, whereas this value for patients with poorly-differentiated gastric cancer is $13.0 \%$. This difference between groups is statistically significant $(P<0.05)$. The relationship between the degree of differentiation and lymph node metastasis in colon cancer is still unclear, which is consistent with our study. There are few studies exploring the relationship between pathological types and lymph node metastasis. Wang [29] found no correlation between pathological types and lymph node metastasis in the study of 103 cases of early gastric cancer. In our research, we found that the pathological nature of the tumor was an independent risk factor for lymph node metastasis in gastric and colon cancer patients $(P=0.021)$, however, the subdivision into gastric and colon cancer patients was not statistically significant. It is considered that the number of early cancer cases in our study is relatively small, and the determination of the type of pathology is mostly dependent on the subjective judgment of endoscopists and surgeons. In some cases, the pathological type is inconsistent with that observed after postoperative pathology, which may be attributed to the experience of clinicians and their judgment of intraoperative lesions.

Based on these differences, we attempted to identify independent risk factors for lymph node metastasis in patients with gastric and colon cancers. In order to increase the accuracy of predicting lymph node metastasis preoperatively, multivariate logistic regression analyses were performed by combining serum characteristics and clinicopathological characteristics. These parameters have rarely been considered in previous studies. We determined that CA125, hemoglobin, albumin, tumor size, pathological type, nerve invasion, and vascular invasion were independent risk factors for lymph node metastasis in gastric and colon cancers. Finally, the preoperative scores, including the above independent risk factors was established. Subsequently, ROC analyses were performed to verify the diagnostic accuracy of the preoperative scores. As expected, the AUC values of Score in lymph node metastasis of gastric and colon cancers were 0.923 and 0.870 , respectively. Its sensitivity was $92.2 \%$, specificity was $78.9 \%$ and $60.2 \%$, respectively, thereby significantly improving the accuracy. Therefore, we believe that the preoperative scores has potential diagnostic value for lymph node metastasis in gastric and colon cancers.

We used the ROC curve to further determine the cutoff value of Score for lymph node metastasis in gastric and colon cancers, and established it to be 287. Accordingly, patients were divided into a high Score group ( $\geq 287$ ) and a low Score group $(<287)$. Further studies revealed that Score was firmly related to several tumor characteristics. A higher Score was associated with a larger tumor size, a certain pathological type, increased differentiation, a deeper degree of invasion, and a more severe lymph node metastasis. Therefore, we believe that the cutoff value of 287 for Score is ideal for the preoperative prediction of lymph node metastasis in gastric and colon cancers. This preoperative scores includes serum indicators and clinicopathological features to make it more comprehensive and accurate. In addition, all involved indicators can be confirmed by preoperative blood tests and preoperative endoscopic examination, which are convenient, inexpensive, and suitable for all patients. Combined with clinical practicality and accuracy, the preoperative scores can be used as a reliable indicator for the development of preoperative surgical plans and comprehensive treatment regimens. 
Our study still has some limitations that should not be neglected. Since this was a retrospective study, tumor-characteristic parameters such as invasion depth, lymphatic invasion, and pathological type were obtained postoperatively. These parameters can be obtained preoperatively by endoscopy, however, the results may be inaccurate. Furthermore, because of the incomplete data and a partial loss of our followup data, we did not add the analysis of prognosis in our study. Moreover, all patients analyzed in our study were from a single hospital. Therefore, our findings still require validation using extensive prospective multicenter studies. Most importantly, the sensitivity of our model was not sufficiently high to diagnose lymph node metastasis. Therefore, it can only be used as an auxiliary tool.

\section{Conclusion}

This is the first study of its kind to explore the relationship between the preoperative scores and lymph node metastasis in gastric and colon cancers. We found that the preoperative scores was independently associated with lymph node metastasis, which significantly improved diagnostic accuracy and could be used to predict lymph node metastases, so as to personalize individual treatments. Additionally, this method is an economical and a convenient tool for predicting lymph node metastasis.

\section{Declarations}

\section{Acknowledgments}

Not applicable

\section{Author Contributions}

X.S conceived and designed the study. M.L and CM.L collected the sample.XJ.Z and XD.C performed the experiments. M.L and XD.C analyzed and interpreted the data. XJ.Z wrote the manuscript and X.S revised the manuscript. All authors discussed the results and implications of the study.

\section{Funding}

Not applicable

\section{Availability of data and materials}

Not applicable

\section{Ethics approval and consent to participate}

The study was approved by the Hospital Ethics Committee

\section{Consent for publication}

Not applicable 


\section{Competing interests}

The authors declare that they have no competing interests.

\section{Author details}

${ }^{1}$ Department of General Surgery, The Dingli Clinical Institute of Wenzhou Medical University (Wenzhou Central Hospital), Wenzhou, Zhejiang, People's Republic of China. ${ }^{2}$ Department of Gastrointestinal Surgery, The Second Affiliated Hospital, Wenzhou Medical University, Wenzhou, Zhejiang, People's Republic of China ; ${ }^{3}$ Department of Gynaecology and Obstetrics, The Dingli Clinical Institute of Wenzhou Medical University (Wenzhou Central Hospital), Wenzhou, Zhejiang, People's Republic of China.

\section{References}

1. Bray F, \en JS, Masuyer E, et al. Estimates of global cancer prevalence for 27 sites in the adult population in 2008囚J囚. Int J Cancer. 2013; 132( 5) : 1133-1145.

2. Tsai HL, Cheng KI, Lu CY, et al. Prognostic significance of depth of invasion, vascular invasion and numbers of lymph node retrievals in combination for patients with stage II colorectal cancer undergoing radical resection $₫ \mathrm{~J} \bigotimes$. J Surg Oncol.2008;97 ( 5) : 383囚387.

3. Chen CY, Wu CW, Lo SS, et al. Peritoneal carcinomatosis and lymph node metastasis are prognostic indicators in patients with Borrmann type IV gastric carcino \J\.Hepatogastroenterology.2002; 49 ( 45) : 874-877.

4. Fleming FJ, Hayanga AJ, Glynn F, et al. Incidence and prognostic influence of lymph node micrometastases in rectal cancer囚J区. Eur J Surg Oncol.2007; 33( 8) : 998 \ 1002.

5. Koh DM, Brown G, Husband JE. Nodal staging in rectal cancer囚J囚. Abdom Imaging. 2006; 31(6): 652-659.

6. Choi IJ.Endoscopic gastric cancer screening and surveillance in high-risk groups.Clin Endosc.2014;47:497-503.

7. Kim J H, Jun $\mathrm{K} \mathrm{H}$, Jung $\mathrm{H}$, et al. Prognostic value of preoperative serum levels of five tumor markers(carcinoembryonic antigen, CA199, alpha -fetoprotein, CA724, and CA125) in gastric cancer [J]. Hepatogastroenterology. 2014; 61(131): 863.

8. Woo Jin Choi, BSc, Michelle C. Cleghorn, et al. Preoperative neutrophil-to -lymphocyte 
ratio is a better prognostic serum biomarker than platelet-to- lymphocyte ratio in patients undergoing resection for nonmetastatic colorectal cancer】J】. Ann Surg Oncol.2015; 10 ( 5) : 125-135.

9. Vinuela EF, Gonen M, Brennan MF, et al. Laparoscopic versus open distal gastrectomy for gastric cancer: a meta- analysis ofrandomized controlled trials and high- quality nonrandomized

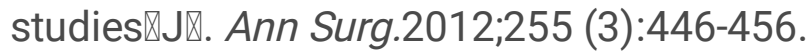

10. Colon Cancer Laparoscopic or Open Resection Study Group, Buunen M,Veldkamp R,et al.Survival after laparoscopic surgery versus open surgery for colon cancer: long-term outcome of a randomised clinical trial囚J囚.Lancet Oncol. 2009; 10 (1) : 44-52.

11. Yu P, Hao Y.The present situation and future development for laparoscopic surgery for gastric cancer in China[J].Zhonghua Wai Ke Za Zhi.2016; 54(1):2-5.

12. Cunningham D, Allum WH, Stenning SP, et al. Perioperative chemotherapy versus surgery alone for resectable gastroesophageal cancer囚J囚. N Engl J Med. 2006; 355(1):11-20.

13. Ychou M, Boige V, Pignon JP, et al. Perioperative chemotherapy compared with surgery alone for resectable gastroesophageal adenocarcinoma: an FNCLCC and FFCD multicenter phase III trial \J囚. J Clin Oncol.2011;29(13):1715-1721.

14. Bunting DM,Lai WW,Berrisford RG,et al. Positron emission tomography-computed tomography in oesophageal cancer staging:a tailored approach. World J Surg.2015; 39: 1000-1007.

15. Liu J, Geng Q, Chen S,et al. Nomogram based on systemic inflammatory response markers predicting the survival of patients with resectable gastric cancer after D2 gastrectomy. Oncotarget. 2016;7(25):37556-37565.

16. Stotz M, Liegl-Atzwanger B, Posch F, et al. Blood-Based Biomarkers Are Associated with Disease Recurrence and Survival in Gastrointestinal Stroma Tumor Patients after Surgical Resection. PLoS One. 2016; 11(7): e0159448.

17. Li YW, Zhang YC.Retrospective study of lymph node metastasis and pathological characteristics of gastric cancer [J].Chinese Journal of Bases and Clinics in General Surgery.2017;24(5):572-579.

18. $\mathrm{Li} \mathrm{HJ}, \mathrm{Che} \mathrm{XM}, \mathrm{He} \mathrm{SC}$, et al.Analysis of risk factors about lymph node metastasis and 
prognosis in colon cancer[J].MODERN ONCOLOGY.2011;19(12):2489-2491.

19. Scartozzi M, Cascinu S. Lymphatic, blood vessel and perineural invasion identifies early

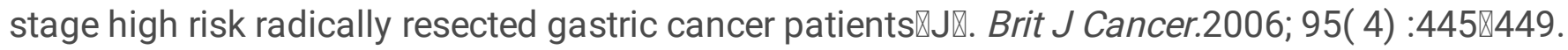

20. Gresta LT, Rodrigues-Junior IA, de Castro LP, Cassali GD, Cabral MM. Assessment of vascular invasion in gastric cancer: a comparative study. World J Gastroenterol. 2013;19:3761-3769.

21. Betge J, Pollheimer MJ, Lindtner RA, et al. Intramural and extramural vascular Invasion in colorectal cancer: prognostic significance and quality of pathology reporting[J]. Cancer.2012;118(3): 628-638

22. Yang YC, Huang XZ , Sun JX, et al.Prognostic Value of Perineural Invasion in Colorectal Cancer: A Meta-Analysis[J]. Gastrointest Surg.2015;19(6):1113-1122.

23. Deng J, You Q, Gao Y, et al. Prognostic value of perineural invasion in gastric cancer: a systematic review and meta- analysis[J]. PLoS One.2014; 9(2):e88907.

24. Tanaka A, Yoshikawa $\mathrm{H}$, Okuno K,et al. The importance of neural invasion (NI) as a prognostic factor in diffuse invasive gastric cancer. Surg Today. 1997;27(8):692-695.

25. Tsai MK, Jeng JY, Lee WJ,et al. Adenocarcinoma of the gastric cardia: prognostic signifificance of pathologic and treatment factors. J Formos Med Assoc. 1995;94(9): 535-540.

26. Ren G, Cai R, Zhang WJ, et al. Prediction of risk factors for lymph node metastasis in early gastric cancer. World J Gastroenterol.2013;19(20): 3096-3107.

27. Bravo Neto GP, dos Santos EG, Victer FC, et al. Lymph node metastasis in early gastric cancer囚J囚. Dev Col Bras Cir.2014; 41 ( 1) : 11-17.

28. Kodera $Y$, Yamamura $Y$, Kanemitsu Y, et al. Lymph node metastasis in cancer of the middle-third stomach: criteria for treatment with a pylorus-preserving gastrectomy. Surg Today. 2001;31(3): 196-203.

29. Wang JM,Zhou LY,Lin SR,et al.Analysis of lymph node metastasis in early gastric cancer and its influencing factors $\llbracket$ J\. Zhonghua Nei Ke Za Zhi.2010; 49( 4) : 297-300. 


\section{Tables}

Table 1: Clinical and pathological characteristics

\begin{tabular}{|ll|}
\hline Variable & 768 \\
\hline $\mathrm{N}$ & $66(58-75)$ \\
\hline Age (years), median (IQR) & $510 / 258$ \\
\hline Sex (male/female) & $22.33(20.20-24.22)$ \\
\hline BMI (kg/m²), median (IQR) & $4.12(2.50-5.00)$ \\
\hline Tumor size (cm), median (IQR) & \\
\hline Tumor location & $312(40.3 \%)$ \\
Gastric cancer & $462(59.7 \%)$ \\
Colon cancer & \\
\hline Pathological type [n, (\%)] & $564(73.4 \%)$ \\
Ulcerative & $204(26.6 \%)$ \\
Non-ulcerative & \\
\hline Histopathological differentiation [n (\%)] & $573(74.6 \%)$ \\
Well-differentiated & $195(25.4 \%)$ \\
Poorly differentiated & $237(30.9 \%)$ \\
\hline Depth of invasion [n (\%)] & $531(69.1 \%)$ \\
T1/T2 & $378(49.6 \%)$ \\
\hline T3/T4 & \\
\hline Lymph node metastasis [n (\%)] & \\
Yes & \\
\hline No & \\
\hline
\end{tabular}

Abbreviations:IQR, interquartile range;BMl,body mass index. 
Table 2: Relationship between preoperative serum markers and lymph node metastasis in patients with gastric and colon cancers

\begin{tabular}{|c|c|c|c|c|}
\hline Factors & Total & LMPG & LMNG & $\begin{array}{l}\mathrm{P}- \\
\text { value }\end{array}$ \\
\hline $\mathrm{AFP}(\mathrm{g} / \mathrm{L})$ & $2.60(2.00-3.60)$ & $2.60(1.93-3.60)$ & $2.60(2.00-3.60)$ & 0.921 \\
\hline $\operatorname{CEA}(\mathrm{g} / \mathrm{L})$ & $2.80(1.60-7.30)$ & $3.10(1.60-7.40)$ & $2.65(1.70-6.70)$ & 0.687 \\
\hline CA125(U/L) & $10.10(7.15-15.95)$ & $11.20(8.00-18.70)$ & $9.40(6.70-13.40)$ & $0.008 *$ \\
\hline CA199(U/L) & $9.20(5.00-16.60)$ & $9.45(4.88-21.83)$ & $8.80(5.1014 .50)$ & 0.136 \\
\hline CA153(U/L) & $7.10(5.10-10.20)$ & $7.10(5.10-11.10)$ & $7.15(5.40-9.40)$ & 0.828 \\
\hline WBC $₫ * 10^{9} / \mathrm{L} \rrbracket$ & $6.00(4.80-7.27)$ & $6.20(4.90-7.80)$ & $5.80(4.70-6.95)$ & 0.082 \\
\hline $\begin{array}{l}\text { Neutrophil } \\
\square^{\star} 10^{9} / \mathrm{L} \rrbracket\end{array}$ & $3.60(2.73-4.80)$ & $3.70(2.80-5.30)$ & $3.40(2.65-4.55)$ & $0.045^{\star}$ \\
\hline $\begin{array}{l}\text { Lymphocyte } \\
\square^{\star} 10^{9} / \text { L】 }\end{array}$ & $1.60(1.20-2.00)$ & $1.50(1.00-1.85)$ & $1.70(1.20-2.00)$ & 0.066 \\
\hline Hemoglobin(g/L) & $\begin{array}{l}125.00(108.25- \\
138.00)\end{array}$ & $\begin{array}{l}121.00(98.50- \\
135.00)\end{array}$ & $\begin{array}{l}128.00(117.00- \\
141.50)\end{array}$ & $0.001 *$ \\
\hline $\mathrm{RBC}\left(* 10^{12} / \mathrm{L}\right)$ & $4.21(3.73-4.70)$ & $4.14(3.56-4.61)$ & $4.27(3.88-4.71)$ & $0.014^{*}$ \\
\hline Platelet $\nabla^{*} 10^{9} / \mathrm{L} \bigotimes$ & $\begin{array}{l}227.50(183.25- \\
281.75)\end{array}$ & $\begin{array}{l}230.00(183.00- \\
312.00)\end{array}$ & $\begin{array}{l}224.00(183.50- \\
264.50)\end{array}$ & 0.174 \\
\hline Albumin(g/L) & $38.15(34.40-40.88)$ & $36.70(33.80-40.15)$ & $38.85(35.73-41.55)$ & $0.004 *$ \\
\hline NLR & $2.21(1.67-3.53)$ & $2.37(1.72-4.14)$ & $2.08(1.63-3.04)$ & $0.015^{\star}$ \\
\hline PLR & $\begin{array}{l}145.62(112.50- \\
201.58)\end{array}$ & $\begin{array}{l}167.14(113.88- \\
226.46)\end{array}$ & $\begin{array}{l}136.00(110.34- \\
179.62)\end{array}$ & $0.014^{\star}$ \\
\hline
\end{tabular}

Notes: * Statistically significant $(P<0.05)$. The values of the variables are presented as the median $(I Q R)$.

Abbreviations:IQR, interquartile range;AFP,alpha fetoprotein; CEA, carcinoembryonic antigen;CA-125, carbohydrate antigen 125;CA-199, carbohydrate antigen 199;CA-153, carbohydrate antigen 153; WBC, white blood cell; RBC, red blood cell;NLR, neutrophil-to-lymphocyte ratio; PLR, platelet-to-lymphocyte ratio; LMPG, lymph node metastasis positive group. LMNG, lymph node metastasis negative group. 
Table 3: Univariate analysis of the risk of lymph node metastasis

\begin{tabular}{|c|c|c|c|c|}
\hline Factors & LMPG (n=384) & LMNG $(n=378)$ & 2 & P-value \\
\hline Sex & & & 0.096 & 0.757 \\
\hline Men & 252 & 255 & & \\
\hline Women & 132 & 123 & & \\
\hline Age (years) & & & 0.244 & 0.621 \\
\hline$<60 y$ & 108 & 117 & & \\
\hline$\geqq 60 y$ & 276 & 261 & & \\
\hline $\mathrm{BMI}\left(\mathrm{kg} / \mathrm{m}^{2}\right)$ & & & 0.700 & 0.403 \\
\hline$<24$ & 312 & 291 & & \\
\hline$\geqq 24$ & 72 & 87 & & \\
\hline Tumor Size $(\mathrm{cm} \rrbracket$ & & & 24.314 & $0.000 *$ \\
\hline$<1.65$ & 3 & 72 & & \\
\hline$\geqq 1.65$ & 381 & 306 & & \\
\hline Pathological type & & & 7.731 & $0.005^{\star}$ \\
\hline Ulcerative & 312 & 249 & & \\
\hline Non-ulcerative & 72 & 129 & & \\
\hline Histopathological differentiation & & & 16.781 & $0.000 *$ \\
\hline Well-differentiated & 243 & 324 & & \\
\hline Poorly-differentiated & 141 & 54 & & \\
\hline Depth of invasion & & & 33.602 & $0.000^{*}$ \\
\hline $\mathrm{T} 1 / 2$ & 54 & 180 & & \\
\hline T3/4 & 330 & 198 & & \\
\hline S100 & & & 52.612 & $0.000 *$ \\
\hline Negative & 165 & 327 & & \\
\hline Positive & 219 & 51 & & \\
\hline CD31/D2-40 & & & 90.513 & $0.000 *$ \\
\hline Negative & 120 & 339 & & \\
\hline Positive & 264 & 39 & & \\
\hline MRP3 & & & 0.767 & 0.381 \\
\hline
\end{tabular}




\begin{tabular}{|lllll|} 
Negative & 251 & 237 & & \\
Positive & 133 & 141 & 0.570 & 0.450 \\
EGFR & 199 & 229 & & \\
Negative & 185 & 149 & & \\
Positive & & & 0.578 & 0.188 \\
P53 & 128 & 147 & & \\
Negative & 256 & 231 & & \\
Positive & & & 0.001 & 0.970 \\
CDX2 & 17 & 17 & & \\
Negative & 367 & 361 & & \\
Positive & & & & \\
\hline
\end{tabular}

Notes:* Statistically significant $(P<0.05)$.

Abbreviations: LMPG, lymph node metastasis positive group;LMNG, lymph node metastasis negative group;BMI,body mass index; LMPG, lymph node metastasis positive group. LMNG, lymph node metastasis negative group. 
Table 4: Multivariate analysis of lymph node metastasis in gastric cancer and colon cancer

\begin{tabular}{|c|c|c|c|c|}
\hline \multirow[t]{2}{*}{ Factor } & \multicolumn{2}{|l|}{ Gastric cancer } & \multicolumn{2}{|l|}{ Colon cancer } \\
\hline & OR(95\% Cl) & P-value & OR $(95 \% \mathrm{Cl})$ & P-value \\
\hline CA125(U/L) & - & & & \\
\hline$<10.65$ & & & Reference & \\
\hline$\geqq 10.65$ & & & $2.660(1.087-6.509)$ & $0.032^{*}$ \\
\hline Albumin(g/L) & & & - & \\
\hline$<36.8$ & 7.804(1.791-34.003) & $0.006 *$ & & \\
\hline$\geqq 36.8$ & Reference & & & \\
\hline PLR & - & & & \\
\hline$<206.76$ & & & Reference & \\
\hline$\geqq 206.76$ & & & $3.896(1.436-10.572)$ & $0.008^{*}$ \\
\hline Histopathological & & & - & \\
\hline Well-differentiated & Reference & & & \\
\hline Poorly-differentiated & $3.601(0.935-13.869)$ & 0.066 & & \\
\hline Depth of invasion & & & - & \\
\hline $\mathrm{T} 1 / 2$ & Reference & & & \\
\hline T3/4 & $5.982(1.599-22.373)$ & $0.008^{*}$ & & \\
\hline Nerve infiltration & - & & & \\
\hline Negative & & & Reference & \\
\hline Positive & & & $8.103(2.632-24.942)$ & $0.000^{*}$ \\
\hline \multicolumn{5}{|l|}{ Vascular infiltration } \\
\hline Negative & Reference & & Reference & \\
\hline Positive & $29.251(6.665-128.37)$ & $0.000 *$ & $9.177(3.113-27.056)$ & $0.000 *$ \\
\hline
\end{tabular}

Notes:* Statistically significant $(P<0.05)$.

Abbreviations:CA-125, carbohydrate antigen 125;PLR, platelet-to-lymphocyte ratio; $\mathrm{Cl}$, confifidence interval; $\mathrm{OR}$, odds ratio. 
Table 5: Multivariate analysis of lymph node metastasis in gastric and colon

\begin{tabular}{|c|c|c|c|c|}
\hline Factor & OR & $95 \% \mathrm{Cl}$ & P-value* & Score \\
\hline \multicolumn{5}{|l|}{ CA125 (U/L) } \\
\hline$<10.65$ & Reference & & & 0 \\
\hline$\geqq 10.65$ & 2.851 & $1.300-2.654$ & 0.009 & 45 \\
\hline \multicolumn{5}{|l|}{ Albumin (g/L) } \\
\hline$<36.8$ & 2.334 & $1.000-5.450$ & 0.050 & 37 \\
\hline$\geqq 36.8$ & Reference & & & 0 \\
\hline \multicolumn{5}{|c|}{ Hemoglobin $(\mathrm{g} / \mathrm{L})$} \\
\hline$<116.5$ & 3.255 & $1.369-7.738$ & 0.008 & 51 \\
\hline$\geqq 116.5$ & Reference & & & 0 \\
\hline \multicolumn{5}{|c|}{ Pathological type } \\
\hline Ulcerative & 2.828 & $1.171-6.830$ & 0.021 & 45 \\
\hline Non-ulcerative & Reference & & & 0 \\
\hline \multicolumn{5}{|c|}{ Tumor size (cm) } \\
\hline$<1.65$ & Reference & & & 0 \\
\hline$\geqq 1.65$ & 15.847 & $1.709-146.909$ & 0.015 & 120 \\
\hline \multicolumn{5}{|c|}{ Nerve infiltration } \\
\hline Negative & Reference & & & 0 \\
\hline Positive & 2.812 & $1.209-6.536$ & 0.016 & 45 \\
\hline \multicolumn{5}{|c|}{ Vascular infiltration } \\
\hline Negative & Reference & & & 0 \\
\hline Positive & 18.200 & $7.217-45.894$ & 0.000 & 126 \\
\hline
\end{tabular}

Notes:*All values in this column are statistically signifificant $(P<0.05)$.

Abbreviations:CA-125, carbohydrate antigen $125 ; \mathrm{Cl}$, confifidence interval; OR, odds ratio. 
Table 6: Clinicopathologic characteristics of gastric and colon cancer patients, based on Score

\begin{tabular}{|c|c|c|c|c|}
\hline Factor & Score $<287$ (432) & Score $\geqq 287$ (336) & 2 & P-value \\
\hline Sex & & & 0.810 & 0.368 \\
\hline Men & 297 & 213 & & \\
\hline Women & 135 & 123 & & \\
\hline Age (years) & & & 0.385 & 0.535 \\
\hline$<60 y$ & 135 & 93 & & \\
\hline$\geqq 60 y$ & 297 & 243 & & \\
\hline CA125 (U/L) & & & 11.136 & $0.001 *$ \\
\hline$<10.65$ & 271 & 137 & & \\
\hline$\geqq 10.65$ & 161 & 199 & & \\
\hline Hemoglobin $(\mathrm{g} / \mathrm{L})$ & & & 9.207 & $0.002 *$ \\
\hline$<116.5$ & 111 & 147 & & \\
\hline$\geqq 116.5$ & 321 & 189 & & \\
\hline Albumin $(\mathrm{g} / \mathrm{L})$ & & & 2.498 & 0.114 \\
\hline$<36.8$ & 162 & 159 & & \\
\hline$\geqq 36.8$ & 270 & 177 & & \\
\hline Pathological type & & & 20.185 & $0.000 *$ \\
\hline Ulcerative & 270 & 294 & & \\
\hline Non-ulcerative & 162 & 42 & & \\
\hline Histopathological & & & 28.871 & $0.000 *$ \\
\hline Well-differentiated & 378 & 195 & & \\
\hline Poorly-differentiated & 54 & 141 & & \\
\hline Tumor size (cm) & & & 21.871 & $0.000 *$ \\
\hline$<1.65$ & 76 & 0 & & \\
\hline$\geqq 1.65$ & 356 & 336 & & \\
\hline Depth of invasion & & & 20.408 & $0.000 *$ \\
\hline $\mathrm{T} 1 / 2$ & 183 & 54 & & \\
\hline $\mathrm{T} 3 / 4$ & 249 & 282 & & \\
\hline
\end{tabular}




\begin{tabular}{|c|c|c|c|c|}
\hline Nerve infiltration & & & 76.307 & $0.000 *$ \\
\hline Negative & 378 & 117 & & \\
\hline Positive & 54 & 219 & & \\
\hline Vascular infiltration & & & 181.663 & $0.000 *$ \\
\hline Negative & 417 & 45 & & \\
\hline Positive & 15 & 291 & & \\
\hline Positive lymph node & & & 91.024 & 0.000 * \\
\hline$<4$ & 420 & 150 & & \\
\hline$\geqq 4$ & 12 & 186 & & \\
\hline
\end{tabular}

Notes:*Statistically signifificant $(P<0.05)$. The values in the table are the number of patients. Abbreviations:CA- 125, carbohydrate antigen-125.

\section{Figures}




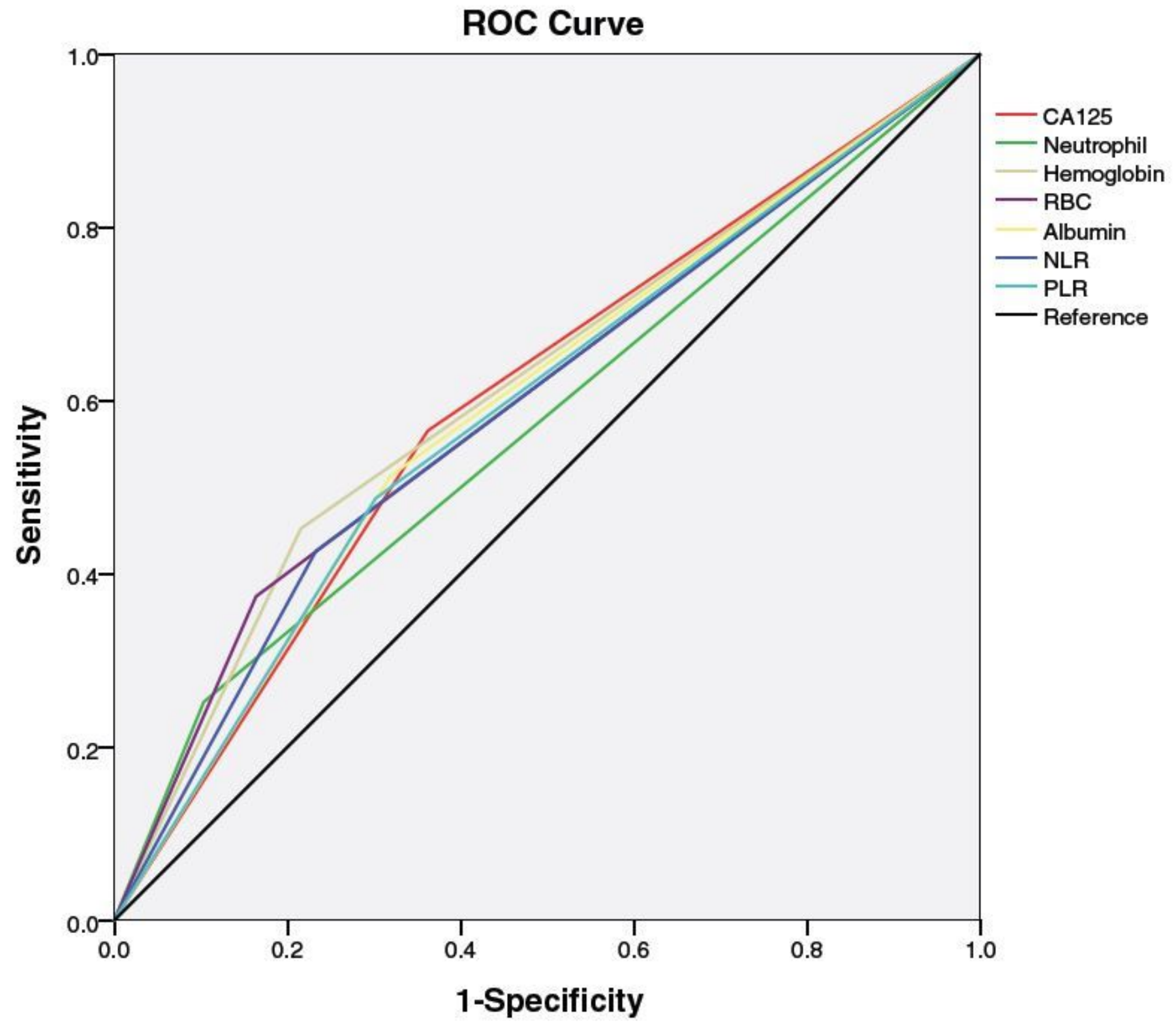

Figure 1

ROC curve for preoperative serum markers in patients with gastric and colon cancers according to lymph node metastasis. 


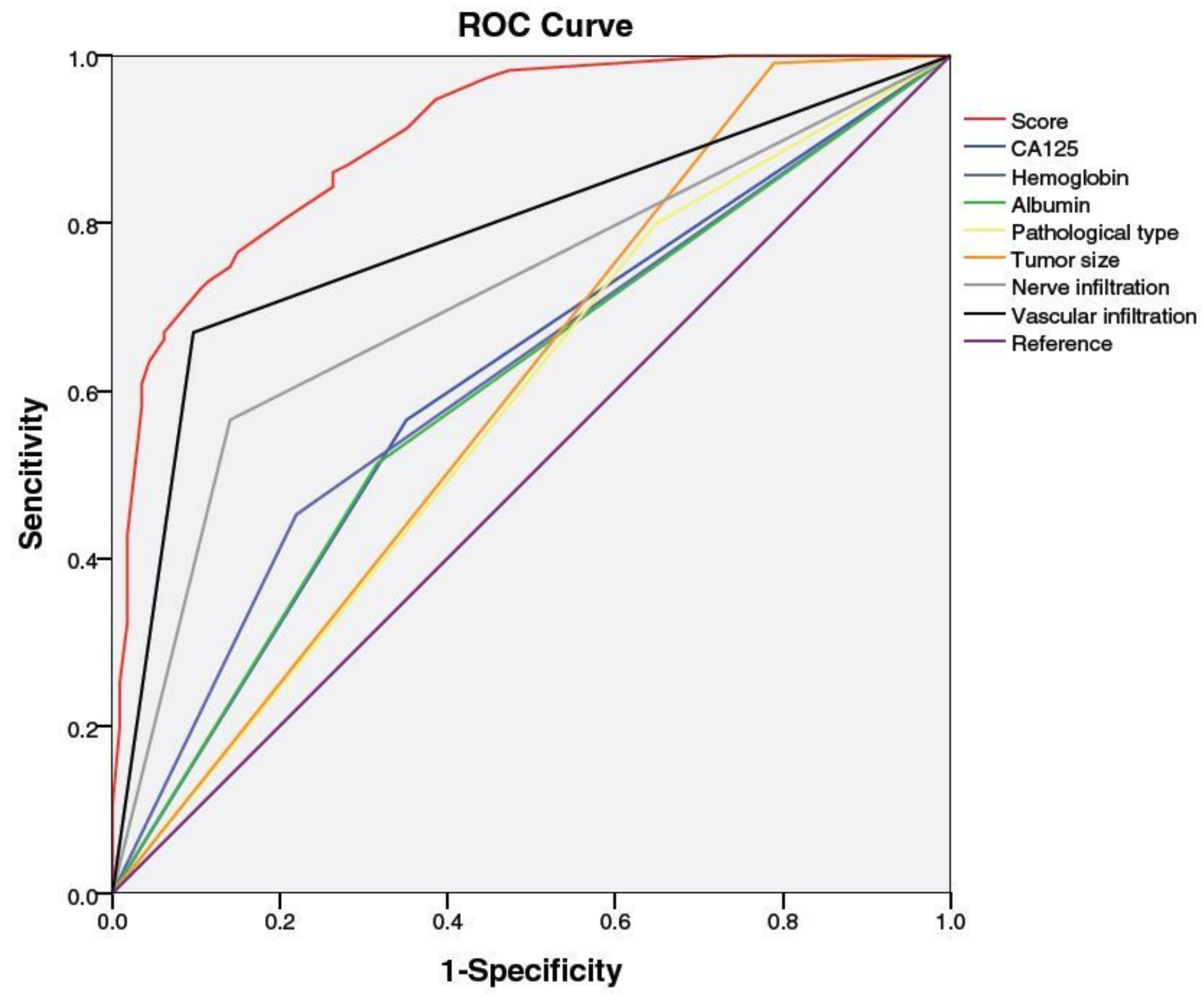

Figure 2

Comparison of ROC curves for Score and the independent risk factors of gastric and colon cancer to evaluate the probability of lymph node metastasis. 


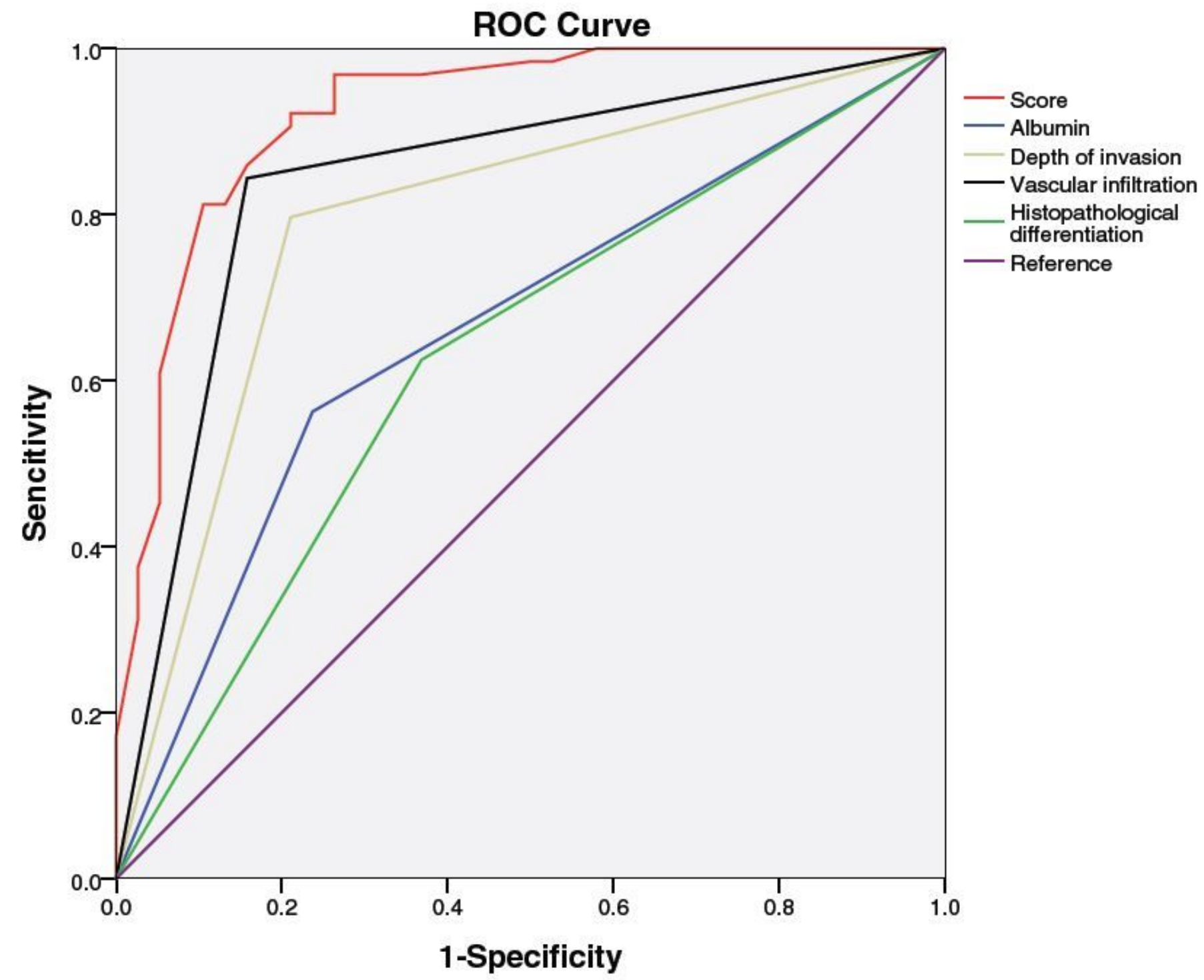

Figure 3

Comparison of ROC curves for Score and the independent risk factors of gastric cancer to evaluate the probability of lymph node metastasis. 


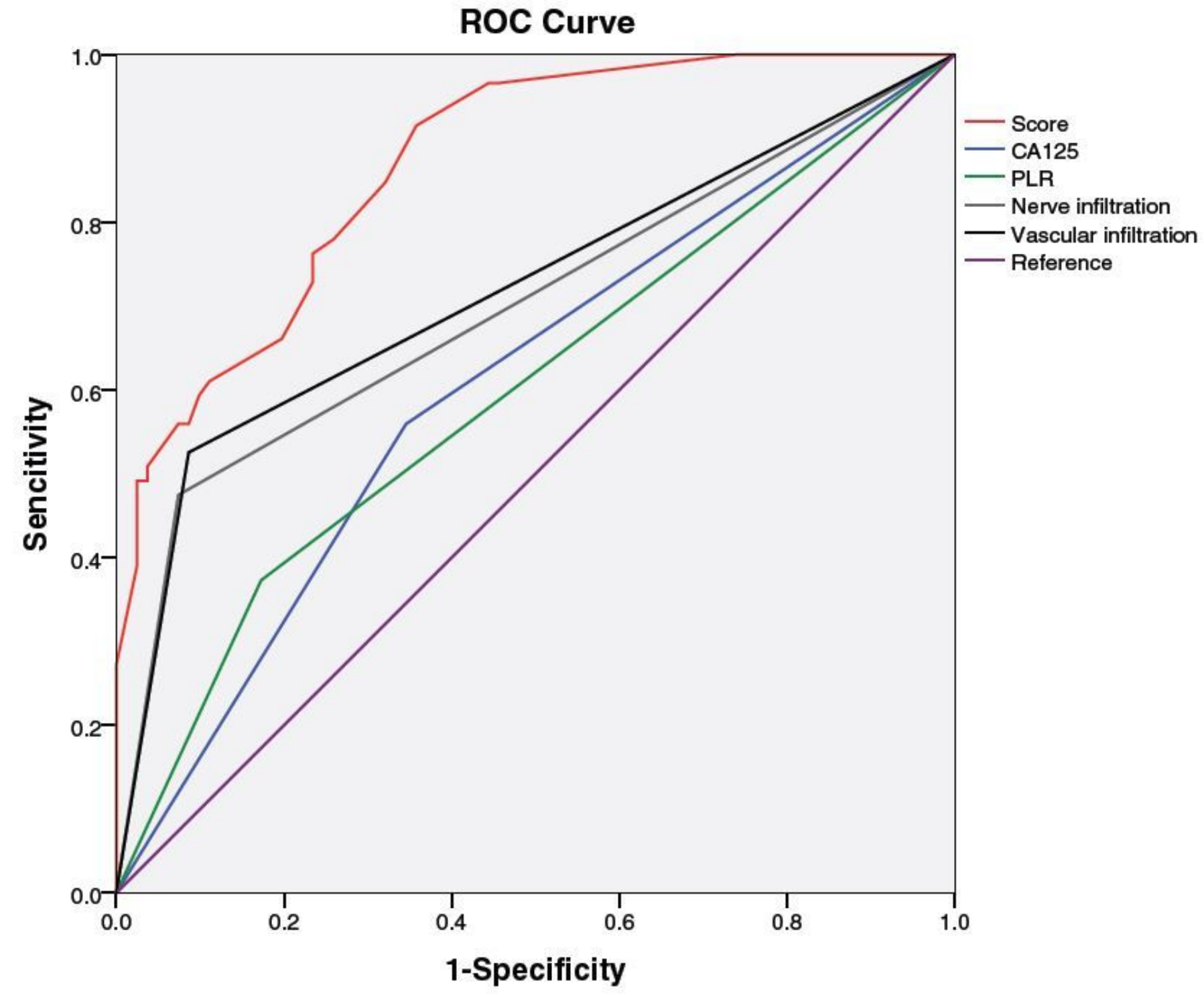

Figure 4

Comparison of ROC curves for Score and the independent risk factors of colon cancer to evaluate the probability of lymph node metastasis. 